\title{
FINITE SEMILATTICES WITH MANY CONGRUENCES
}

\author{
GÁBOR CZÉDLI
}

\begin{abstract}
For an integer $n \geq 2$, let $\operatorname{NCSL}(n)$ denote the set of sizes of congruence lattices of $n$-element semilattices. We find the four largest numbers belonging to $\operatorname{NCSL}(n)$, provided that $n$ is large enough to ensure that $|\operatorname{NCSL}(n)| \geq 4$. Furthermore, we describe the $n$-element semilattices witnessing these numbers.
\end{abstract}

\section{INTRODUCTION AND MOTIVATION}

The present paper is primarily motivated by a problem on tolerance relations of lattices raised by Joanna Grygiel in her conference talk in September, 2017, which was a continuation of Górnicka, Grygiel, and Tyrala [5]. Further motivation is supplied by Czédli [1], Czédli and Mureşan [2], Kulin and Mureşan [8], and Mureşan [9], still dealing with lattices rather than semilattices.

As usual, $\operatorname{Con}(A)$ will stand for the lattice of congruences of an algebra $A$. Given a natural number $n \geq 2$ and a variety $\mathcal{V}$ of algebras, the task of

finding the small numbers in the set $\mathrm{NC}(\mathcal{V}, n):=$ $\{|\operatorname{Con}(A)|: A \in \mathcal{V}$ and $|A|=n\}$ and describing the

algebras $\mathcal{V}$ witnessing these numbers

has already deserved some attention for various varieties $\mathcal{V}$, because the description of the simple $n$-element algebras in $\mathcal{V}$ for various varieties $\mathcal{V}$ and, in particular, even the Classification of Finite Simple Groups belong to (1.1) in some vague sense. The present paper addresses an analogous problem, which is obtained from (1.1) by changing "small" to "large". Of course, this problem is hopeless for an arbitrary variety $\mathcal{V}$. However, if $\mathcal{V}$ is the variety SLat $\wedge$ of meet-semilattices, see Remark 2.8 for this terminology, then we can benefit from Freese and Nation's classical description of the congruence lattices of finite members of SLat $\wedge$; see [4]. Let us fix the following notation

$$
\operatorname{NCSL}(n):=\operatorname{NC}\left(\operatorname{SLat}_{\wedge}, n\right)=\left\{|\operatorname{Con}(S)|: S \in \operatorname{SLat}_{\wedge} \text { and }|S|=n\right\} ;
$$

the acronym NCSL comes from "Number of Congruences of SemiLattices". Our target is to determine the four largest numbers belonging to $\operatorname{NCSL}(n)$ and, in addition, to describe the $n$-element semilattices witnessing these numbers.

1991 Mathematics Subject Classification. 06A12, secondary 06B10 finite lattice, lattice with many congruences.

This research was supported by the Hungarian Research Grant KH 126581. 
Outline. The rest of the paper is structured as follows. In Section 2, we introduce a semilattice construction, and we use this construction in formulating the main result, Theorem 2.3, to realize our target mentioned above. This section concludes with a corollary stating that a semilattice with sufficiently many congruences is planar. Section 3 is devoted to the proof of Theorem 2.3.

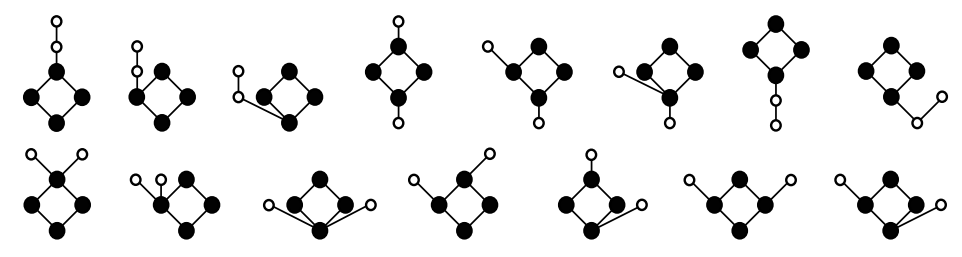

Figure 1 . The full list of 6 -element meet-semilattices with exactly $28=28 \cdot 2^{6-6}$ many congruences

\section{QuASI-TREE SEMILATTICES AND OUR THEOREM}

We follow the standard terminology and notation; see, for example, Grätzer [6] and [7]. In particular, $a \| b$ means that $a$ and $b$ are incomparable, that is, neither $a \leq b$, nor $b \leq a$. Even without explicitly saying so all the time, by a semilattice we always mean a finite meet semilattice $S$, that is, a finite member of SLat ${ }_{\wedge}$. Such an $S=\langle S ; \wedge\rangle$ has a least element $0=\bigwedge S$. We always denote $S \backslash\{0\}$ by $S^{+}$. Note that $\vee$, denoting supremum with respect to the ordering inherited from $\langle S ; \wedge\rangle$, is only a partial operation and $\left\langle S^{+} ; \vee\right\rangle$ is a partial algebra in general. If no two incomparable elements of $S$ have an upper bound, then $S$ is called a tree semilattice.

Next, for a meet-semilattice $S$, the congruence $\boldsymbol{\tau}=\boldsymbol{\tau}(S ; \wedge)$ generated by

$$
\left\{\langle a \wedge b, a \vee b\rangle: a, b \in S^{+}, a \| b \text {, and } a \vee b \text { exists in }\left\langle S^{+} ; \vee\right\rangle\right\}
$$

will be called the tree congruence of $\langle S ; \wedge\rangle$. Of course, we can write $a, b \in S$ instead of $a, b \in S^{+}$above. Observe that for $a, b \in S^{+}$,

$$
\{a, b\} \text { has an upper bound in } S \text { iff } a \vee b \text { exists in }\left\langle S^{+} ; \vee\right\rangle \text {; }
$$

hence instead of requiring the join $a \vee b \in\left\langle S^{+} ; \vee\right\rangle$, it suffices to require an upper bound of $a$ and $b$ in (2.1). The name "tree congruence" is explained by the following easy statement, which will be proved in Section 3 .
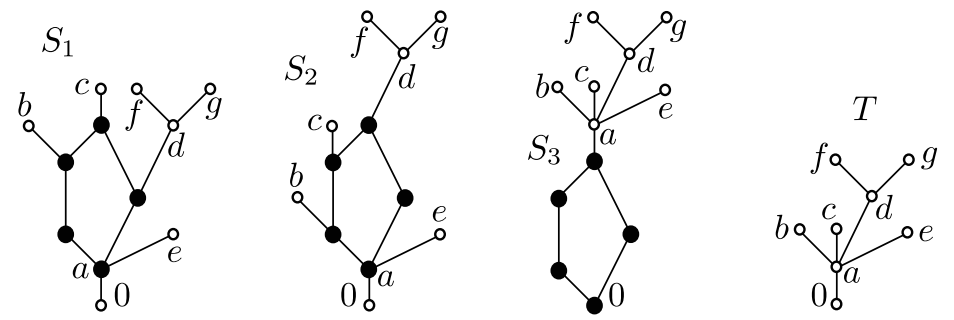

Figure 2. Three twelve-element meet-semilattices with the same skeleton $T$ and the same number, $26 \cdot 2^{12-6}=1664$, of congruences

Proposition 2.1. For an arbitrary finite meet-semilattice $\langle S ; \wedge\rangle$, the quotient meetsemilattice $\langle S ; \wedge\rangle / \tau$ is a tree. 
Definition 2.2. By a quasi-tree semilattice we mean a finite meet-semilattice $\langle S ; \wedge\rangle$ such that its tree congruence $\boldsymbol{\tau}=\boldsymbol{\tau}(S ; \wedge)$ has exactly one nonsingleton block. If $\langle S ; \wedge\rangle$ is a quasi-tree semilattice, then the unique nonsingleton block of $\boldsymbol{\tau}$, which is a meet-semilattice, and the quotient semilattice $\langle S ; \wedge\rangle / \tau$ are called the nucleus and the skeleton of $\langle S ; \wedge\rangle$.

Some quasi-tree semilattices are shown in Figures 1, 2, and 3. In these figures, the elements of the nuclei are the black-filled ones, while the empty-filled smaller circles stand for the rest of elements. Although a quasi-tree semilattice $\langle S ; \wedge\rangle$ is not determined by its skeleton and nucleus in general, the skeleton and the nucleus together carry a lot of information on $\langle S ; \wedge\rangle$. In order to make the numbers occurring in the following theorem easy to compare, we give them in a redundant way as multiples of $2^{n-6}$.

Theorem 2.3. If $\langle S ; \wedge\rangle$ is a finite meet-semilattice of size $n=|S|>1$, then the following hold.

(i) $\langle S ; \wedge\rangle$ has at most $2^{n-1}=32 \cdot 2^{n-6}$ many congruences. Furthermore, we have that $|\operatorname{Con}(S ; \wedge)|=2^{n-1}$ if and only if $\langle S ; \wedge\rangle$ is a tree semilattice.

(ii) If $\langle S ; \wedge\rangle$ has less than $2^{n-1}=32 \cdot 2^{n-6}$ congruences, then it has at most $28 \cdot 2^{n-6}$ congruences. Furthermore, $|\operatorname{Con}(S ; \wedge)|=28 \cdot 2^{n-6}$ if and only if $\langle S ; \wedge\rangle$ is a quasi-tree semilattice and its nucleus is the four-element boolean lattice; see Figure 1 for $n=6$.

(iii) If $\langle S ; \wedge\rangle$ has less than $28 \cdot 2^{n-6}$ congruences, then it has at most $26 \cdot 2^{n-6}$ congruences. Furthermore, $|\operatorname{Con}(S ; \wedge)|=26 \cdot 2^{n-6}$ if and only if $\langle S ; \wedge\rangle$ is a quasi-tree semilattice such that its nucleus is the pentagon $N_{5}$; see Figure 4 and $S_{1}, \ldots, S_{3}$ in Figure 2.

(iv) If $\langle S ; \wedge\rangle$ has less than $26 \cdot 2^{n-6}$ congruences, then it has at most $25 \cdot 2^{n-6}$ congruences. Furthermore, $|\operatorname{Con}(S ; \wedge)|=25 \cdot 2^{n-6}$ if and only if $\langle S ; \wedge\rangle$ is a quasi-tree semilattice such that its nucleus is either $F$, or $N_{6}$; see Figure 4 and $S_{4}, \ldots, S_{7}$ in Figure 3.

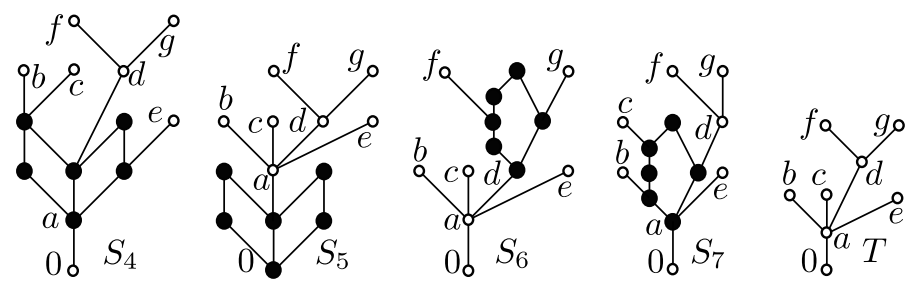

Figure 3. Four thirteen-element meet-semilattices with the same skeleton $T$ and the same number, $25 \cdot 2^{13-6}=3200$, of congruences

Remark 2.4. Although Theorem 2.3 holds for all $n \geq 2$, it neither gives the four largest numbers of $\operatorname{NCSL}(n)$, nor does it say too much for $n \leq 5$. For example, $25 \cdot 2^{n-6}$ is not even an integer if $n \leq 5$. Hence, we note the following facts without including their trivial proofs in the paper.

(A) $\operatorname{NCSL}(2)=\left\{2=2^{2-1}\right\}$

(B) $\operatorname{NCSL}(3)=\left\{4=2^{3-1}\right\}$

(C) $\operatorname{NCSL}(4)=\left\{8=2^{4-1}, 7=28 \cdot 2^{4-6}\right\}$ 
(D) $\operatorname{NCSL}(5)=\left\{16=2^{5-1}, 14=28 \cdot 2^{5-6}, 13=26 \cdot 2^{5-6}, 12\right\}$. Note that 12 is witnessed by $M_{3}=\left\langle M_{3}, \wedge\right\rangle$; see Figure 4 .
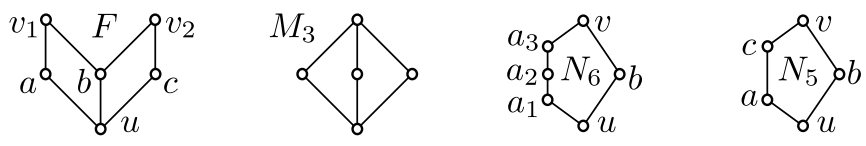

Figure 4. $F, M_{3}, N_{6}$, and the pentagon, $N_{5}$

A semilattice is planar if it has a planar Hasse diagram, that is a Hasse diagram in which edges can intersect only at their endpoints, that is, at vertices. Theorem 2.3 immediately implies the following statement.

Corollary 2.5. If an n-element meet-semilattice has at least $25 \cdot 2^{n-6}$ congruences, then it is planar.

The following statement is due to Freese [3]; see also Czédli [1] for a second proof, which gives the first half of the following corollary for arbitrary finite algebras in congruence distributive varieties, not only for lattices.

Corollary 2.6. For every n-element lattice $L$, we have that $|\operatorname{Con}(L)| \leq 2^{n-1}$. Furthermore, $|\operatorname{Con}(L)|=2^{n-1}$ if and only if $L$ is a chain.

As a preparation for a remark below, we derive this corollary from Theorem 2.3(i) here rather than in the next section.

Proof of Corollary 2.6. The only $n$-element tree semilattice that is also a lattice is the $n$-element chain. For an equivalence relation $\Theta$ on this chain $\langle C ; \leq\rangle$,

$$
\begin{aligned}
& \Theta \in \operatorname{Con}(C ; \wedge) \text { iff } \Theta \in \operatorname{Con}(C ; \vee, \wedge) \text { iff } \\
& \text { every } \Theta \text {-block is an interval of }\langle C ; \leq\rangle .
\end{aligned}
$$

Observe that every $\Theta \in \operatorname{Con}(L ; \vee, \wedge)$ also belongs to $\operatorname{Con}(L ; \wedge)$. Hence, using Theorem 2.3(i) at $\leq^{*}$ below, we obtain that

$$
|\operatorname{Con}(L ; \vee, \wedge)| \leq|\operatorname{Con}(L ; \wedge)| \leq{ }^{*}|\operatorname{Con}(C ; \wedge)|=|\operatorname{Con}(C ; \vee, \wedge)|,
$$

proving Corollary 2.6.

Next, we point out that Theorem 2.3(i) plays an essential role in the proof above.

Remark 2.7. By Szpilrajn's Extension Theorem [10], every (partial) ordering on a set can be extended to a linear ordering. Hence, the second part of (2.3) might give the false feeling that this Extension Theorem in itself implies Corollary 2.6 as follows: extend the ordering relation of $L$ to a linear ordering to obtain a chain; then we obtain more intervals and thus more equivalences whose blocks are intervals, and so more congruences by (2.3). In order to point out that this argument does not work, let $\left\langle L ; \leq_{1}\right\rangle$ be the direct product of the two-element chain and the threeelement chain. Although $\leq_{1}$ can be extended to a linear ordering $\leq_{2}$ and the chain $\left\langle L ; \leq_{2}\right\rangle$ has more intervals than $\left\langle L ; \leq_{1}\right\rangle$, the lattice $\left\langle L ; \leq_{1}\right\rangle$ has 34 equivalences whose blocks are intervals but the chain $\left\langle L ; \leq_{2}\right\rangle$ has only 32 . 
Remark 2.8. The concept of meet-semilattices $\langle S ; \wedge\rangle$ and that of semilattices as commutative and idempotent semigroups $\langle S ; \cdot\rangle$ are well known to be equivalent; see, for example, Grätzer [6, Exercises I.1.41-42 in pp. 18-19]. This paper gives preference to the former approach because of two reasons. First, as opposed to semilattices where there are two natural ways of defining an ordering, it is generally accepted that $a \leq b \Longleftrightarrow a \wedge b=a$ for arbitrary elements $a$ and $b$ of a meet-semilattice $\langle S ; \wedge\rangle$. Second, our figures and many arguments are order theoretical even though congruences are defined in the usual algebraic and semigroup theoretical way.

\section{Proofs}

Proof of Proposition 2.1. A subset $X$ of $\langle S ; \wedge\rangle$ is said to be convex, if $x<y<z$ and $x, z \in X$ imply that $y \in X$, for any $x, y, z \in S$. It is well known that

the blocks of every congruence of $\langle S ; \wedge\rangle$ are convex subsets of $\langle S ; \wedge\rangle$.

Indeed, if $\Theta \in \operatorname{Con}(S ; \wedge), x \leq y \leq z$ and $\langle x, z\rangle \in \Theta$, then $\langle x, y\rangle=\langle x \wedge y, z \wedge y\rangle \in \Theta$, whereby $y \in x / \Theta$, which shows (3.1). By (3.1), the $\boldsymbol{\tau}$-blocks are convex subsets of $\langle S ; \wedge\rangle$. Next, for the sake of contradiction, suppose that $a, b \in S$ such that $a / \tau$ and $b / \tau$ are incomparable elements of the meet-semilattice $\langle S ; \wedge\rangle / \tau$ and they have an upper bound $c / \tau \in\langle S ; \wedge\rangle / \tau$. Let $a^{\prime}:=a \wedge c$ and $b^{\prime}:=b \wedge c$ in $\langle S ; \wedge\rangle$. Since $a / \boldsymbol{\tau} \leq c / \boldsymbol{\tau}$, we have that $a / \boldsymbol{\tau}=a / \boldsymbol{\tau} \wedge c / \boldsymbol{\tau}=(a \wedge c) / \boldsymbol{\tau}=a^{\prime} / \boldsymbol{\tau}$, whence $\left\langle a, a^{\prime}\right\rangle \in \boldsymbol{\tau}$. Similarly, $\left\langle b, b^{\prime}\right\rangle \in \tau$. Since $a^{\prime} \leq c$ and $b^{\prime} \leq c,(2.2)$ implies the existence of $a^{\prime} \vee b^{\prime} \in\left\langle S^{+} ; \vee\right\rangle$. Hence, by the definition of $\boldsymbol{\tau}$, we have that $\left\langle a^{\prime} \wedge b^{\prime}, a^{\prime} \vee b^{\prime}\right\rangle \in \boldsymbol{\tau}$. Since the $\boldsymbol{\tau}$-block $\left(a^{\prime} \wedge b^{\prime}\right) / \boldsymbol{\tau}$ is convex, $\left\langle a^{\prime}, b^{\prime}\right\rangle \in \boldsymbol{\tau}$. Combining this with $\left\langle a, a^{\prime}\right\rangle \in \boldsymbol{\tau}$ and $\left\langle b, b^{\prime}\right\rangle \in \boldsymbol{\tau}$, we obtain that $\langle a, b\rangle \in \boldsymbol{\tau}$. Hence, $a / \boldsymbol{\tau}$ equals $b / \boldsymbol{\tau}$, which contradicts their incomparability.

Note that, in general, $\boldsymbol{\tau}=\boldsymbol{\tau}(S ; \wedge)$ is not the smallest congruence of $\langle S ; \wedge\rangle$ such that $\langle S ; \wedge\rangle / \tau$ is a tree; this is exemplified by the semilattice reduct of the fourelement boolean lattice.

The proof of Theorem 2.3 will be divided into several lemmas, some of them being interesting in themselves, and we are going to prove parts (i)-(iv) separately.

Remember that, for a finite meet-semilattice $S=\langle S ; \wedge\rangle$, we use the notation $S^{+}:=S \backslash\{0\}$. Then $\left\langle S^{+} ; \vee\right\rangle$ is a partial algebra, which we call the partial joinsemilattice associated with $S$. By a partial subalgebra of $\left\langle S^{+} ; \vee\right\rangle$ we mean a subset $X$ of $S^{+}$such that whenever $x, y \in X$ and $x \vee y$ is defined in $\left\langle S^{+} ; \vee\right\rangle$, then $x \vee y \in X$. With respect to the set inclusion relation $\subseteq$, the set of all partial subalgebras of $\left\langle S^{+} ; \vee\right\rangle$ turns out to be a lattice, which we denote by $\operatorname{Sub}\left(S^{+} ; \vee\right)$. For convenience, our convention is that $\emptyset \in \operatorname{Sub}\left(S^{+} ; \vee\right)$. The proof of Theorem 2.3 relies on the following result of Freese and Nation [4].

Lemma 3.1 (Freese and Nation [4, Lemma 1]). For every finite meet-semilattice $\langle S ; \wedge\rangle$, the lattice $\operatorname{Con}(S ; \wedge)$ is dually isomorphic to $\operatorname{Sub}\left(S^{+} ; \vee\right)$. In particular, we have that $|\operatorname{Con}(S ; \wedge)|=\left|\operatorname{Sub}\left(S^{+} ; \vee\right)\right|$.

Note that Freese and Nation [4] uses $\operatorname{Sub}(S ; \vee, 0)$, which does not contain the emptyset, but the natural isomorphism from $\operatorname{Sub}\left(S^{+} ; \vee\right)$ onto $\operatorname{Sub}(S ; \vee, 0)$, defined by $X \mapsto X \cup\{0\}$, allows us to cite their result in the above form. The following lemma is almost trivial; having no reference at hand, we are going to present a short proof. As usual, intervals are nonempty subsets of the form $[a, b]:=\{x: a \leq x \leq b\}$. 
The principal ideal and the principal filter generated by an element $a \in S$ are denoted by $\downarrow a=\{x \in S: x \leq a\}$ and $\uparrow a=\{x \in S: a \leq x\}$, respectively. Meetclosed convex subsets are convex subsemilattices. A subsemilattice is nontrivial if it consists of at least two elements.

Lemma 3.2. Let $X$ be a nontrivial convex subsemilattice of a finite semilattice $\langle S ; \wedge\rangle$, and denote the smallest element of $X$ by $u:=\wedge X$. Then the following two conditions are equivalent.

(a) The equivalence $\Theta$ on $S$ whose only nonsingleton block is $X$ is a congruence of $\langle S ; \wedge\rangle$.

(b) For all $c \in S \backslash \uparrow u$ and every maximal element $v$ of $X$, we have that $u \wedge c=v \wedge c$.

Proof of Lemma 3.2. Assume (a) and let $c \notin \uparrow u$, and let $v$ be a maximal element of $X$. Then $c \notin \uparrow v, u \not \leq \wedge \wedge$, and $u \nless \leq v \wedge c$. Hence, none of $u \wedge c$ and $v \wedge c$ is in $X$, but these two elements are collapsed by $\Theta$ since $\langle u, v\rangle \in \Theta$. Thus, the definition of $\Theta$ gives that $u \wedge c=v \wedge c$, proving that (a) implies (b).

Next, assume (b), and let $\Theta$ be defined as in (a). First, we show that for all $x, y, z \in S$,

$$
\text { if }\langle x, y\rangle \in \Theta \text {, then }\langle x \wedge z, y \wedge z\rangle \in \Theta \text {. }
$$

This is trivial for $x=y$, so we can assume that $x, y \in X$. Pick maximal elements $x_{1}$ and $y_{1}$ in $X$ such that $x \leq x_{1}$ and $y \leq y_{1}$. First, let $z \in \uparrow u$. Then, using the convexity of $X, x \wedge z \in[u, x] \subseteq X$ and, similarly, $y \wedge z \in X$, whence we obtain that $\langle x \wedge z, y \wedge z\rangle \in \Theta$ by the definition of $\Theta$. Second, let $z \in S \backslash \uparrow u$. Then $x \wedge z$ belongs to the interval $\left[u \wedge z, x_{1} \wedge z\right]$, which is the singleton set $\{u \wedge z\}$ by (b). Hence, $x \wedge z=u \wedge z$. Similarly, $y \wedge z=u \wedge z$, whereby $\langle x \wedge z, y \wedge z\rangle \in \Theta$. Thus, (3.2) holds.

Finally, if $\left\langle x_{1}, y_{1}\right\rangle \in \Theta$ and $\left\langle x_{2}, y_{2}\right\rangle \in \Theta$, then we obtain from (3.2) that both $\left\langle x_{1} \wedge x_{2}, y_{1} \wedge x_{2}\right\rangle$ and $\left\langle y_{1} \wedge x_{2}, y_{1} \wedge y_{2}\right\rangle$ belong to $\Theta$, whereby transitivity gives that $\left\langle x_{1} \wedge x_{2}, y_{1} \wedge y_{2}\right\rangle \in \Theta$. Consequently, $\Theta$ is a congruence and (b) implies (a).

The powerset of a set $A$ will be denoted by $P(A)=\{X: X \subseteq A\}$. In the rest of the paper,

$n \geq 2$ denotes a natural number, $\langle S ; \wedge\rangle$ will stand

for an $n$-element meet-semilattice, and we will also use the notation $\quad k:=|\operatorname{Con}(S ; \wedge)|=\left|\operatorname{Sub}\left(S^{+} ; \vee\right)\right|$;

here the second equality is valid by Lemma 3.1.

Proof of Theorem 2.3(i). Since $\left|S^{+}\right|=n-1, S^{+}$has at most $2^{n-1}$ subsets, whereby $|\operatorname{Con}(S ; \wedge)|=k \leq\left|P\left(S^{+}\right)\right|=2^{n-1}$, as required. If $\langle S ; \wedge\rangle$ is a tree semilattice, then $x \vee y$ is defined only if $x$ and $y$ form a comparable pair of $S^{+}$, whence $x \vee y \in\{x, y\}$. Hence, every subset of $S^{+}$belongs to $\operatorname{Sub}\left(S^{+} ; \vee\right)$, and so $k=\left|\operatorname{Sub}\left(S^{+} ; \vee\right)\right|=$ $\left|P\left(S^{+}\right)\right|=2^{n-1}$. If $S$ is not a tree semilattice, then there is a pair $\langle a, b\rangle$ of incomparable elements of $S^{+}$with an upper bound. By (2.2), $a \vee b$ is defined in $\left\langle S^{+} ; \vee\right\rangle$. Hence, $\{a, b\} \notin \operatorname{Sub}\left(S^{+} ; \vee\right)$ and so $k=\left|\operatorname{Sub}\left(S^{+} ; \vee\right)\right|<\left|P\left(S^{+}\right)\right|=2^{n-1}$. This completes the proof of part (i).

By an upper bounded two-element antichain, abbreviated as ubt-antichain, we mean a two-element subset $\{x, y\}$ of a finite meet-semilattice $\langle S ; \wedge\rangle$ such that $x \| y$ and $\uparrow x \cap \uparrow y \neq \emptyset$. By (2.2), every ubt-antichain $\{x, y\}$ has a join in $S^{+}$but this join is outside $\{x, y\}$. Therefore,

$$
\operatorname{Sub}\left(S^{+} ; \vee\right) \text { contains no ubt-antichain. }
$$


Besides (3.4), the importance of ubt-antichains is explained by the following lemma.

Lemma 3.3. Let $X$ be a convex subsemilattice of a finite semilattice $\langle S ; \wedge\rangle$ such that $|X| \geq 2$ and $X \times X \subseteq \boldsymbol{\tau}$; see (2.1). If $X$ contains all ubt-antichains $\{p, q\}$ of $\langle S ; \wedge\rangle$ together with their joins $p \vee q$, then $\langle S ; \wedge\rangle$ is a quasi-tree semilattice and its nucleus is $X$.

Proof of Lemma 3.3. Denote the smallest element of $X$ by $u:=\bigwedge X$. Let $\Theta$ be the equivalence relation on $S$ with $X$ as the only nonsingleton block of $\Theta$. In order to prove that $\Theta \in \operatorname{Con}(S ; \wedge)$, assume that $c \in S \backslash \uparrow u$ and $v$ is a maximal element of $X$. For the sake of contradiction, suppose that $u \wedge c \neq v \wedge c$, which means that $u \wedge c<v \wedge c$. If we had that $v \wedge c \leq u$, then $v \wedge c=u \wedge(v \wedge c)=(u \wedge v) \wedge c=u \wedge c$ would be a contradiction. Thus, $v \wedge c \not \leq u$. On the other hand, $u \notin t v \wedge$ since $u \not \subset c$, whereby $u \| v \wedge c$. Since $v$ is a common upper bound of $u$ and $v \wedge c$, we obtain that $\{u, v \wedge c\}$ is a ubt-antichain. This is a contradiction since $c \notin \uparrow u$ implies that $u \not \leq v \wedge c$, whence the ubt-antichain $\{u, v \wedge c\}$ is not a subset of $X$. Hence, $u \wedge c=v \wedge c$, and it follows from Lemma 3.2 that $\Theta \in \operatorname{Con}(S ; \wedge)$.

Next, in order to show that $\langle S ; \wedge\rangle / \Theta$ is a tree, suppose the contrary. Then there are two incomparable $\Theta$-blocks $x / \Theta$ and $y / \Theta$ that have an upper bound $z / \Theta$. Since $u \in X$ and all other $\Theta$-blocks are singletons, every $\Theta$-block has a smallest element. This fact allows us to assume that each of $x, y$, and $z$ is the least element of its $\Theta$-block. Since $x / \Theta \leq z / \Theta$, we have that $x / \Theta=x / \Theta \wedge z / \Theta=(x \wedge z) / \Theta$, that is, $\langle x, x \wedge z\rangle \in \Theta$. But the least element of $x / \Theta$ is $x$, whence $x=x \wedge z$, that is, $x \leq z$. We obtain similarly that $y \leq z$, that is, $\{x, y\}$ has an upper bound, $z$. Since $x \wedge y=x$ would imply that $x / \Theta \wedge y / \Theta=(x \wedge y) / \Theta=x / \Theta$, contradicting that $\{x / \Theta, y / \Theta\}$ is an antichain, we obtain that $x \not \leq y$. We obtain $y \nless \leq x$ similarly. Thus, $\{x, y\}$ is a ubt-antichain, whereby $\{x, y\} \subseteq X$. But then $x / \Theta=X=y / \Theta$, contradicting the initial assumption that these two $\Theta$-blocks are incomparable. Therefore, $\langle S ; \wedge\rangle / \Theta$ is a tree. Hence, in order to complete the proof, we need to show that $\Theta=\boldsymbol{\tau}$. Since $X \times X \subseteq \boldsymbol{\tau}$, the inclusion $\Theta \subseteq \boldsymbol{\tau}$ is clear. In order to see the converse inclusion, let $\langle a \wedge b, a \vee b\rangle$ be a pair occurring in (2.1). Then $\{a, b\}$ is a ubt-antichain, so $\{a, b\} \subseteq X$ and, by the assumptions of the lemma, both $a \vee b$ and $a \wedge b$ belong to $X$. Hence, the pairs in (2.1) are collapsed by $\Theta$ and we conclude that $\tau \subseteq \Theta$. Consequently, $\Theta=\tau$, and the proof of Lemma 3.3 is complete.

Lemma 3.4. If $\langle S ; \wedge\rangle$ from (3.3) contains exactly one ubt-antichain, then $\langle S ; \wedge\rangle$ is a quasi-tree semilattice and its nucleus is the four-element boolean lattice.

Proof of Lemma 3.4. Let us denote by $\{a, b\}$ the unique ubt-antichain of $\langle S ; \wedge\rangle$. Let $v:=a \vee b$, which exists by (2.2), and let $u:=a \wedge b$. Then $L:=[u, v]$ contains every ubt-antichain. Since $\langle u, v\rangle \in \boldsymbol{\tau}$ by (2.1) and the $\boldsymbol{\tau}$-blocks are convex, $L \times L \subseteq \boldsymbol{\tau}$. So, with reference to Lemma 3.3, it suffices to show that $L$ is the four-element boolean lattice. In fact, it suffices to show that $L \subseteq\{u, a, b, v\}$ since the converse inclusion is evident. Suppose the contrary, and let $x \in L \backslash\{u, a, b, v\}$. If $x \| a$, then $\{a, x\}$ is a ubt-antichain (with upper bound $v$ ) but it is distinct from $\{a, b\}$, which contradicts the fact that $\{a, b\}$ is the only ubt-antichain. Hence, $a$ and $x$ and comparable. We obtain similarly that $b$ and $x$ are comparable. If $x \leq a$ and $x \leq b$, then $u \leq x \leq a \wedge b=u$ leads to $x=u \in L$, which is not the case. We obtain dually that the conjunction of $x \geq a$ and $x \geq b$ is impossible. Hence, $a \leq x \leq b$ or $b \leq x \leq a$, contradicting that $\{a, b\}$ is an antichain. This shows that $L \subseteq\{u, a, b, v\}$, completing the proof of Lemma 3.4. 
Proof of Theorem 2.3(ii). Assume that $k<2^{n-1}$; see (3.3). By Theorem 2.3(i), $\langle S ; \wedge\rangle$ is not a tree. Hence, $n=|S| \geq 4$. Since $\left|\operatorname{Sub}\left(S^{+} ; \vee\right)\right|=k<2^{n-1}=\left|P\left(S^{+}\right)\right|$, not every subset of $S^{+}$is $\vee$-closed. Thus, we can pick $a, b \in S^{+}$such that $a \| b$ and $a \vee b$ exists in $\left\langle S^{+} ; \vee\right\rangle$. Since $\left|S^{+} \backslash\{a, b, a \vee b\}\right|=n-4$, there are $2^{n-4}$ subsets of $S^{+}$that contain $a, b$, but not $a \vee b$; these subsets do not belong to $\operatorname{Sub}\left(S^{+} ; \vee\right)$. Thus, $k \leq 2^{n-1}-2^{n-4}=32 \cdot 2^{n-6}-4 \cdot 2^{n-6}=28 \cdot 2^{n-6}$, proving the first half of (ii).

Next, assume that $k=28 \cdot 2^{n-6}$ and choose $a$ and $b$ as above. There are $2^{n-4}=4 \cdot 2^{n-6}$ subsets of $S^{+}$containing $a$ and $b$, but not containing $a \vee b$; these subsets are not in $\left\langle S^{+} ; \vee\right\rangle$. Thus, all the remaining $32 \cdot 2^{n-6}-4 \cdot 2^{n-6}=28 \cdot 2^{n-6}$ subsets belong to $\left\langle S^{+} ; \vee\right\rangle$ since $k=28 \cdot 2^{n-6}$. In particular, for every ubt-antichain $\{x, y\}$, we have that $\{x, y\} \neq\{a, b\} \Rightarrow\{x, y\} \in \operatorname{Sub}\left(S^{+} ; \vee\right)$. This implication and (3.4) yield that $\{a, b\}$ is the only ubt-antichain in $\langle S ; \wedge\rangle$. Thus, it follows from Lemma 3.4 that $\langle S ; \wedge\rangle$ is a quasi-tree semilattice of the required form.

Conversely, assume that $\langle S ; \wedge\rangle$ is of the form described in Theorem 2.3(ii). Choosing the notation so that its nucleus is $\{a \wedge b, a, b, a \vee b\}$, the only ubtantichain is $\{a, b\}$, whence a subset $X$ of $S^{+}$is not in $\operatorname{Sub}\left(S^{+} ; \vee\right)$ iff $a, b \in X$ but $a \vee b \notin X$. There are $2^{n-4}=4 \cdot 2^{n-6}$ such subsets $X$, and we obtain that $k=\left|\operatorname{Sub}\left(S^{+} ; \vee\right)\right|=\left|P\left(S^{+}\right)\right|-4 \cdot 2^{n-6}=32 \cdot 2^{n-6}-4 \cdot 2^{n-6}=28 \cdot 2^{n-6}$, as required. This completes the proof of Theorem 2.3(ii).

Lemma 3.5. If $\langle S ; \wedge\rangle$ from (3.3) contains exactly two ubt-antichains, $\{a, b\}$ and $\{c, b\}$ such that $a\langle c$, then $\langle S ; \wedge\rangle$ is a quasi-tree semilattice and its nucleus is the pentagon lattice $N_{5}$.

Proof of Lemma 3.5. By (2.2), we can let $v:=a \vee b$. Since $v \leq c$ would lead to $b \leq c$, we have that $v \not \leq c$. In particular, $v \neq c$, and we also have that $v \notin\{a, b\}$ since $\{a, b\}$ is an antichain. Thus, $\{c, v\}$ is a two-element subset of $S$ and it is distinct from $\{a, b\}$ and $\{a, c\}$. Hence, $\{c, v\}$ is not a ubt-antichain. Since $b \vee c$, which exists by (2.2), is clearly an upper bound of $\{c, v\}$, it follows that $\{c, v\}$ is not an antichain. This fact and $v \leq c$ yield that $c \leq v$. Thus, $v=a \vee b \leq c \vee b \leq v$, that is, $v=a \vee b=c \vee b$. Next, let $u:=b \wedge c$; clearly, $u \notin\{b, c\}$. If we had that $a \| u$, then $\{a, u\}$ would be a third ubt-antichain (with upper bound $c$ ), whence $a$ and $u$ are comparable elements. Since $a \leq u$ would lead to $a \leq b$ by transitivity, we have that $u \leq a$. Hence, $u \leq a \wedge b \leq c \wedge b=u$, and so $a \wedge b=u$. The equalities established so far show that $L:=\{u, a, b, c, v\}$ is a sublattice isomorphic to $N_{5}$. In order to show that $L$ is the interval $[u, v]$, suppose the contrary, and let $x \in[u, v] \backslash L$. If $x \| b$, then $\{b, x\}$ would be a third ubt-antichain (with upper bound $v$ ), which would be a contradiction. If we had that $b<x<v$, then $\{c, x\}$ would be a ubt-antichain, a contradiction. Similarly, $u<x<b$ gives that $\{a, x\}$ is a ubt-antichain, a contradiction again. Thus, $L=[u, v]$ is an interval of $S$. By (2.1), $\langle u, v\rangle=\langle a \wedge b, a \vee b\rangle \in \boldsymbol{\tau}$. Using that the $\boldsymbol{\tau}$-blocks are convex subsets, we obtain that $L \times L=[u, v] \times[u, v] \subseteq \boldsymbol{\tau}$. Thus, Lemma 3.5 follows from Lemma 3.3.

Proof of Theorem 2.3(iii). Assume that $k<28 \cdot 2^{n-6}$; see (3.3).

Note at this point that no equality will be assumed for $k$ before (3.24). Therefore the numbered equations, equalities, and statements before (3.24) can be used later in the proof of Theorem 2.3(iv).

We introduce the following notation. For a ubt-antichain $\{a, b\}$, let

$$
U(a, b):=\left\{X \in P\left(S^{+}\right): a \in X, b \in X, \text { but } a \vee b \notin X\right\} ;
$$


it is a subset of $P\left(S^{+}\right)$; note that the existence of $a \vee b$ above follows from (2.2). By Theorem 2.3(i), $\langle S ; \wedge\rangle$ is not a tree, whereby it has at least one ubt-antichain. If it had only one ubt-antichain, then Lemma 3.4 and Theorem 2.3(ii) would imply that $k=28 \cdot 2^{n-6}$. Hence, $\langle S ; \wedge\rangle$ has at least two ubt-antichains. Let $\left\{a_{1}, b_{1}\right\},\left\{a_{2}, b_{2}\right\}$, $\ldots,\left\{a_{t}, b_{t}\right\}$ be a repetition-free list of all ubt-antichains of $\langle S ; \wedge\rangle$; note that $t \geq 2$. Let $v_{i}:=a_{i} \vee b_{i}$ and $U_{i}:=U\left(a_{i}, b_{i}\right)$, see (3.5), for $i=1, \ldots, t$. That is, $U_{i}$ is the set of all those $X \in P\left(S^{+}\right)$that contain $a_{i}$ and $b_{i}$ but not $v_{i}$. Observe that, for $1 \leq i<j \leq t$,

$$
\text { if }\left|\left\{a_{i}, b_{i}, v_{i}, a_{j}, b_{j}, v_{j}\right\}\right|=\ell \text {, then }\left|U_{i} \cap U_{j}\right| \text { is either } 2^{5-\ell} \cdot 2^{n-6} \text {, or } 0 .
$$

Indeed, when we choose elements from the $(n-1)$-element $P\left(S^{+}\right)$in order to form a set $X \in U_{i} \cap U_{j}$, then we can dispose only over $(n-1)-\ell=(5-\ell)+(n-6)$ elements, because the containment $X \in U_{i} \cap U_{j}$ determines what to do with $\ell$ elements. If the stipulations for these $\ell$ elements are contradictory, then $\left|U_{i} \cap U_{j}\right|$ equals 0 ; this can happen only if $\ell<6$. Otherwise, $\left|U_{i} \cap U_{j}\right|=2^{5-\ell} \cdot 2^{n-6}$, showing the validity of (3.6).

Next, we show that for any $1 \leq i<j \leq t$,

$$
\begin{aligned}
& \text { if }\left|\left\{a_{i}, b_{i}, v_{i}, a_{j}, b_{j}, v_{j}\right\}\right|=6 \text {, then } k \leq 24.5 \cdot 2^{n-6}, \\
& \text { if }\left|\left\{a_{i}, b_{i}, v_{i}, a_{j}, b_{j}, v_{j}\right\}\right|=5 \text {, then } k \leq 25 \cdot 2^{n-6}, \text { and } \\
& \text { if }\left|\left\{a_{i}, b_{i}, v_{i}, a_{j}, b_{j}, v_{j}\right\}\right|=4 \text {, then } k \leq 26 \cdot 2^{n-6} \text {. }
\end{aligned}
$$

As a stronger form of (3.4) for the present situation, it is clear that

$$
\operatorname{Sub}\left(S^{+} ; \vee\right)=P\left(S^{+}\right) \backslash\left(U_{1} \cup \cdots \cup U_{t}\right) .
$$

In particular, $U_{i} \cup U_{j}$ is disjoint from $\operatorname{Sub}\left(S^{+} ; \vee\right)$. Hence, the Inclusion-Exclusion Principle, $k=\left|\operatorname{Sub}\left(S^{+} ; \vee\right)\right|,\left|P\left(S^{+}\right)\right|=32 \cdot 2^{n-6}$, and $\left|U_{i}\right|=\left|U_{j}\right|=4 \cdot 2^{n-6}$ give that

$$
\begin{aligned}
\operatorname{Sub}\left(S^{+} ; \vee\right) & \subseteq P\left(S^{+}\right) \backslash\left(U_{i} \cup U_{j}\right), \text { and so } \\
k & \leq 2^{n-6} \cdot(32-4-4)+\left|U_{i} \cap U_{j}\right|=24 \cdot 2^{n-6}+\left|U_{i} \cap U_{j}\right|,
\end{aligned}
$$

and if (3.11) holds with equality in it, then so does (3.12).

Clearly, (3.7), (3.8), and (3.9) follow from (3.6) and (3.12). Furthermore, it is also clear from this argument that strict inequalities lead to strict inequalities. For later reference, we formulate this as follows.

If $\left|U_{i} \cap U_{j}\right|$ is strictly less than $2^{n-7}, 2^{n-6}$, and $2 \cdot 2^{n-6}$, then $k$ is strictly less than $24.5 \cdot 2^{n-6}$, $25 \cdot 2^{n-6}$, and $26 \cdot 2^{n-6}$, respectively.

Next, we claim that for $1 \leq i<j \leq t$,

$$
\text { if } v_{i} \neq v_{j} \text {, then }\left|\left\{a_{i}, b_{i}, v_{i}, a_{j}, b_{j}, v_{j}\right\}\right| \geq 5 \text {. }
$$

In order to show this, first we deal with the case where $v_{j} \in\left\{a_{i}, b_{i}\right\}$ or $v_{i} \in\left\{a_{j}, b_{j}\right\}$. Let, say, $v_{1}=a_{2}$. Then $v_{2}>a_{2}=v_{1}>a_{1}$ and $v_{2}>a_{2}=v_{1}>b_{1}$ yield that $\left|\left\{a_{1}, b_{1}, v_{1}, v_{2}\right\}\right|=4$. Clearly, $b_{2} \notin\left\{a_{2}=v_{1}, v_{2}\right\}$. If we had that $b_{2} \in\left\{a_{1}, b_{1}\right\}$, then $b_{2}<v_{1}=a_{2}$ would contradict $a_{2} \| b_{2}$. Hence, the inequality in (3.15) holds in this case. Second, assume that $v_{j} \notin\left\{a_{i}, b_{i}\right\}$ and $v_{i} \notin\left\{a_{j}, b_{j}\right\}$. Using also that $v_{i} \neq v_{j}$, we have that $\left|\left\{a_{i}, b_{i}, v_{i}, v_{j}\right\}\right|=4$. Since $v_{i} \notin\left\{a_{j}, b_{j}\right\},\left\{a_{i}, b_{i}\right\} \neq\left\{a_{j}, b_{j}\right\}$, 
and, of course, $v_{j} \notin\left\{a_{j}, b_{j}\right\}$, at least one of $a_{j}$ and $b_{j}$ is not in $\left\{a_{i}, b_{i}, v_{i}, v_{j}\right\}$, and the required inequality in (3.15) holds again. This proves (3.15). Clearly,

$$
\text { if } v_{i}=v_{j} \text { but } i \neq j \text {, then }\left|\left\{a_{i}, b_{i}, v_{i}, a_{j}, b_{j}, v_{j}\right\}\right| \geq 4 \text {, }
$$

because $\left\{a_{i}, b_{i}, a_{j}, b_{j}\right\}$ has at least three elements and does not contain $v_{i}=v_{j}$, which is strictly larger than every element of $\left\{a_{i}, b_{i}, a_{j}, b_{j}\right\}$. Observe that the inequality $k \leq 26 \cdot 2^{n-6}$, which is the first half of Theorem 2.3(iii), follows from (3.7), (3.8), (3.9), (3.15), and (3.16), because $t \geq 2$ implies the existence of a pair $\langle i, j\rangle$ such that $1 \leq i<j \leq t$.

Next, strengthening (3.8), we are going to show that for any $1 \leq i<j \leq t$,

$$
\text { if }\left|\left\{a_{i}, b_{i}, v_{i}, a_{j}, b_{j}, v_{j}\right\}\right|=5 \text { and } t \geq 3 \text {, then } k<25 \cdot 2^{n-6} \text {. }
$$

Assume the premise of (3.17). Since $t \geq 3$, we can pick an $m \in\{1, \ldots, t\} \backslash\{i, j\}$. For the sake of contradiction,

$$
\text { suppose that }\left|\left\{a_{i}, b_{i}, v_{i}, a_{j}, b_{j}, v_{j}\right\}\right|=5 \text { but } k \geq 25 \cdot 2^{n-6} \text {. }
$$

By (3.6) and (3.18), $\left|U_{i} \cap U_{j}\right|$ is either 0 or $2^{n-6}$, but the first alternative is ruled out by (3.14) and (3.18). Hence

$$
\left|U_{i} \cap U_{j}\right|=2^{n-6} .
$$

By (3.7) and (3.18), none of $\left\{a_{i}, b_{i}, v_{i}, a_{m}, b_{m}, v_{m}\right\}$ and $\left\{a_{j}, b_{j}, v_{j}, a_{m}, b_{m}, v_{m}\right\}$ consists of six elements. Hence, it follows from (3.15) and (3.16), that each of these two sets consists of four or five elements. Thus, (3.6) gives that

$$
\left|U_{i} \cap U_{m}\right| \leq 2 \cdot 2^{n-6} \text { and }\left|U_{j} \cap U_{m}\right| \leq 2 \cdot 2^{n-6} .
$$

We also need the following observation.

$$
\begin{aligned}
& \text { If } U_{i} \cap U_{j} \neq \emptyset, U_{i} \cap U_{m} \neq \emptyset, \text { and } U_{j} \cap U_{m} \neq \emptyset, \\
& \text { then } U_{i} \cap U_{j} \cap U_{m} \neq \emptyset .
\end{aligned}
$$

To show (3.21), assume that its premise holds. If $\left\{a_{i}, b_{i}, a_{j}, b_{j}, a_{m}, b_{m}\right\}$ is disjoint from $\left\{v_{i}, v_{j}, v_{m}\right\}$, then $U_{i} \cap U_{j} \cap U_{m}$ contains $\left\{a_{i}, b_{i}, a_{j}, b_{j}, a_{m}, b_{m}\right\}$ and so it is nonempty. Otherwise, by $a-b$ symmetry and since the subscripts in (3.21) play symmetric roles, we can assume that $a_{i}=v_{j}$. However, then $U_{i} \cap U_{j}=\emptyset$, contradicting the premise of (3.21). Consequently, (3.21) holds. Based on the Inclusion-Exclusion Principle, as in (3.11)-(3.13), and using (3.19) and (3.20), we can compute as follows; the overline and the underlines below will serve as reference points.

$$
\begin{aligned}
k \leq 2^{n-6} \cdot(32-(4+4+4))+ & +\left(\left|U_{i} \cap U_{j}\right|+\left|U_{i} \cap U_{m}\right|+\left|U_{j} \cap U_{j}\right|\right) \\
& -\left|U_{i} \cap U_{j} \cap U_{m}\right|, \quad \text { and so } \\
k \leq & 2^{n-6} \cdot(20+\overline{1}+\underline{2}+\underline{2})-\left|U_{i} \cap U_{j} \cap U_{m}\right| \\
= & 25 \cdot 2^{n-6}-\left|U_{i} \cap U_{j} \cap U_{m}\right| .
\end{aligned}
$$

We know from (3.19) that $U_{i} \cap U_{j} \neq \emptyset$. Both underlined numbers in (3.23) come from (3.20). So if at least one the intersections $U_{i} \cap U_{m}$ and $U_{j} \cap U_{m}$ is empty, then at least one of the underlined numbers can be replaced by 0 and (3.23) gives that $k<25 \cdot 2^{n-6}$. Otherwise the subtrahend at the end of (3.23) is positive by (3.21), and we obtain again that $k<25 \cdot 2^{n-6}$.

This contradicts (3.18) and proves the validity of (3.17). Next, we assume that

$$
k=26 \cdot 2^{n-6} \text {. }
$$


It follows from (3.7), (3.8), (3.15), and (3.24) that

all the $v_{i}$ are the same, so we can let $v:=v_{1}=\cdots=v_{t}$.

Hence, we get from (3.6), (3.7), (3.8), (3.16), and (3.24) that, for any $1 \leq i<j \leq t$,

$$
\begin{aligned}
& \left|\left\{a_{i}, b_{i}, a_{j}, b_{j}, v\right\}\right|=4 \text { and so }\left|U_{i} \cap U_{j}\right| \leq 2 \cdot 2^{n-6}, \\
& \left|\left\{a_{i}, b_{i}, a_{j}, b_{j}\right\}\right|=3 \text {, and }\left|\left\{a_{i}, b_{i}\right\} \cap\left\{a_{j}, b_{j}\right\}\right|=1 .
\end{aligned}
$$

Next, we are going to prove that $t$, the number of ubt-antichains, equals 2 . Suppose the contrary. Since now we have (3.26) instead of (3.19), $\overline{1}$ and 25 in (3.23) turn into $\underline{2}$ and 26 , respectively. These two modifications do not influence the paragraph following (3.23), and we conclude that the inequality in the modified (3.23) is a strict one, that is, $k<26 \cdot 2^{n-6}$. This contradicts (3.24), whence we conclude that there are exactly $t=2$ ubt-antichains. We know from (3.26) that they are not disjoint. So we can denote them by $\{a, b\}$ and $\{c, b\}$ where $|\{a, b, c\}|=3$. By (3.25), $v=a \vee b=c \vee b$. Since $t=2$, the set $\{a, c\}$ is not a ubt-antichain, whence $a$ and $c$ are comparable. So we can assume that $a<c$, and it follows from Lemma 3.5 that $\langle S ; \wedge\rangle$ is a quasi-tree semilattice of the required form.

Finally, assume that $\langle S ; \wedge\rangle$ is a quasi-tree semilattice and its nucleus is the pentagon $N_{5}=\{u, a, b, c, v\}$ with bottom $u$, top $v$, and $a<c$. Let $U_{1}:=U(a, b)$ and $U_{2}:=U(c, b)$; see (3.5). Since $\operatorname{Sub}\left(S^{+} ; \vee\right)=P\left(S^{+}\right) \backslash\left(U_{1} \cup U_{2}\right)$ by (3.10),

$$
k=\left|P\left(S^{+}\right)\right|-\left|U_{1}\right|-\left|U_{2}\right|+\left|U_{1} \cap U_{2}\right|=(32-4-4+2) \cdot 2^{n-6}=26 \cdot 2^{n-6},
$$

as required. This completes the proof of Theorem 2.3(iii).

Lemma 3.6. If $\langle S ; \wedge\rangle$ from (3.3) contains exactly two ubt-antichains, $\{a, b\}$ and $\{b, c\}$ such that $v_{1}:=a \vee b$ and $v_{2}:=b \vee c$ are incomparable, then $\langle S ; \wedge\rangle$ is a quasi-tree semilattice and its nucleus is $F=\left\{u:=a \wedge b \wedge c, a, b, c, v_{1}, v_{2}\right\}$ given in Figure 4.

Proof of Lemma 3.6. Let $u:=a \wedge b$. It is not in $\{a, b\}$. Since $b \nsupseteq c$, we have that $u \ngtr c$. Using that $v_{2}$ is an upper bound of $\{u, c\}$ and $\{u, c\}$ is not a ubt-antichain, it follows that $\{u, c\}$ is not an antichain. Hence, $u \leq c$, whence $u=a \wedge b \wedge c$. Since $a \| b$ and $v_{1} \| v_{2}$ implies that $a \| c$, we obtain that $a \wedge c \notin\{a, b, c\}$. Hence, $\{b, a \wedge c\}$ is a two-element set and it is distinct from $\{a, b\}$ and $\{b, c\}$. Using that $v_{1}$ is an upper bound of $\{b, a \wedge c\}$, we obtain that $\{b, a \wedge c\}$ is not an antichain. Since $b \not \leq c$, we have that $b \not \leq a \wedge c$. Hence, $a \wedge c \leq b$, implying that $a \wedge c=a \wedge c \wedge b$. Summarizing the facts above and taking into account that $a$ and $c$ play a symmetric role, we have that

$$
u=a \wedge b=a \wedge b \wedge c=b \wedge c=a \wedge c .
$$

Let $M:=\left\{a, b, c, u, v_{1}, v_{2}\right\}$; we claim that

$M$ is a convex meet-subsemilattice of $\langle S ; \wedge\rangle$.

First, we show that $M$ is a convex subset of $\langle S ; \wedge\rangle$. For the sake of contradiction, suppose that $x \in S \backslash M$ such that $u<x<v_{1}$; the case $u<x<v_{2}$ would be similar since $a$ and $c$ play symmetric roles. Both $\{a, x\}$ and $\{x, b\}$ have an upper bound, $v_{1}$. Hence, none of them is an ubt-antichain since $x \notin M$. Thus, $a \leq x \leq b$, or $b \leq x \leq a$, or $a, b \in \downarrow x$, or $a, b \in \uparrow x$. The first two alternatives are ruled out by $a \| b$. The third alternative leads to $v_{1}=a \vee b \leq x \leq v_{1}$, contradicting $x \notin M$. We obtain a contradiction from the fourth alternative dually by using $u$ instead of $v_{1}$. Thus, $M$ is a convex subset of $\langle S ; \wedge\rangle$. Since $M$ is convex and 
$b \leq v_{1} \wedge v_{2} \leq v_{1}$, we have that $v_{1} \wedge v_{2} \in\left\{b, v_{1}\right\}$. Similarly, $v_{1} \wedge v_{2} \in\left\{b, v_{2}\right\}$. So $v_{1} \wedge v_{2} \in\left\{b, v_{1}\right\} \cap\left\{b, v_{2}\right\}=\{b\}$, that is, $v_{1} \wedge v_{2}=b$. This equality together with (3.27) give easily that $M$ is a meet-subsemilattice of $\langle S ; \wedge\rangle$, whence (3.28) holds. It is clear by $(3.27)$ that $M \cong F$.

Since $\left\langle u, v_{1}\right\rangle=\langle a \wedge b, a \vee b\rangle$ occurs in (2.1) and the $\boldsymbol{\tau}$-blocks are convex subsets, $\left\{a, b, v_{1}, u\right\} \subseteq u / \boldsymbol{\tau}$. We obtain similarly that $\left\{b, c, v_{2}, u\right\} \subseteq u / \boldsymbol{\tau}$, whence we have that $M \times M \subseteq \tau$. Therefore, since $M$ contains both ubt-antichains and their joins, Lemma 3.3 implies the validity of Lemma 3.6.

Lemma 3.7. If $\langle S ; \wedge\rangle$ from (3.3) contains exactly three ubt-antichains, $\left\{a_{1}, b\right\}$, $\left\{a_{2}, b\right\}$, and $\left\{a_{3}, b\right\}$ such that $v:=a_{1} \vee b=a_{2} \vee b=a_{3} \vee b$ and $a_{1}<a_{2}<a_{3}$, then $\langle S ; \wedge\rangle$ is a quasi-tree semilattice and its nucleus is $N_{6}=\left\{u:=a_{1} \wedge b=a_{2} \wedge b=\right.$ $\left.a_{3} \wedge b, a_{1}, a_{2}, a_{3}, v\right\}$ given in Figure 4.

Proof of Lemma 3.7. Let $u:=a_{3} \wedge b$. Since $a_{3} \| b, u \neq b$. We are going to show that $M:=\left\{u, a_{1}, a_{2}, a_{3}, v\right\}$ is a subsemilattice isomorphic to $N_{6}$. Let $i \in\{1,2\}$. Since $v$ is an upper bound of the set $\left\{a_{i}, u\right\}$, this set is not an antichain. Since $a_{i} \not \leq b$, we have that $a_{i} \not \leq u$. Hence, $u<a_{i}$, and we obtain that $u \leq a_{i} \wedge b \leq a_{3} \wedge b=u$. Thus, the meets in $M$ are what they are required to be, and we conclude that $M \cong N_{6}$. Next, for the sake of contradiction, suppose that $M$ is not a convex subset of $\langle S ; \wedge\rangle$, and pick an element $x \in S \backslash M$ such that $u \leq x \leq v$. Since no more ubt-antichain is possible, none of $a_{1}, a_{2}, a_{3}$, and $b$ is incomparable with $x$. If we had that $x \leq a_{j}$ for some $j \in\{1,2,3\}$, then $b \leq x$ would contradict $b \not \leq a_{j}$ while $x \leq b$ would lead to $u \leq x \leq a_{j} \wedge b \leq u$, a contradiction since $x \neq u \in M$. A dual argument, with $v$ instead of $u$, would lead to a contradiction if $a_{j} \leq x$. Hence, $M$ is a convex subsemilattice of $\langle S ; \wedge\rangle$. Since $\langle u, v\rangle=\left\langle a_{1} \wedge b, a_{1} \vee b\right\rangle$ occurs in (2.1) and the $\boldsymbol{\tau}$ blocks are convex subsets, $M \times M \subseteq \tau$. Therefore, since $M$ contains all the three ubt-antichains and their common join, Lemma 3.7 follows from Lemma 3.3.

Proof of Theorem 2.3(iv). We assume that $k=|\operatorname{Con}(S ; \wedge)|<26 \cdot 2^{n-6}$. In the first part of the proof, we are going to focus on the required inequality, $k \leq 25 \cdot 2^{n-6}$.

As it has been mentioned in the proof of Theorem 2.3(iii), any part of that proof before (3.24) is applicable here, including the notation. If $\left|\left\{a_{i}, b_{i}, v_{i}, a_{j}, b_{j}, v_{j}\right\}\right| \geq 5$ or $v_{i} \neq v_{j}$ for some $1 \leq i<j \leq t$, then the required $k \leq 25 \cdot 2^{n-6}$ follows from (3.7), (3.8), and (3.15). Hence, we can assume that $v:=v_{1}=v_{2}=\cdots=v_{t}$. By (3.8) and (3.16), we can assume also that $\left|\left\{a_{i}, b_{i}, a_{j}, b_{j}, v\right\}\right|=4$ for all $1 \leq i<j \leq t$. For later reference, we summarize our assumptions as

$$
\begin{aligned}
& v:=v_{1}=v_{2}=\cdots=v_{t} \text { and }\left|\left\{a_{i}, b_{i}, a_{j}, b_{j}, v\right\}\right|=4 \text {, whereby } \\
& \left|\left\{a_{i}, b_{i}, a_{j}, b_{j}\right\}\right|=3 \text { and }\left|\left\{a_{i}, b_{i}\right\} \cap\left\{a_{j}, b_{j}\right\}\right|=1 \text {, for all } 1 \leq i<j \leq t .
\end{aligned}
$$

We claim that

$$
\begin{aligned}
& \text { if } t \geq 3,(3.29) \text {, and }\left\{a_{1}, b_{1}\right\} \cap\left\{a_{2}, b_{2}\right\} \cap\left\{a_{3}, b_{3}\right\}=\emptyset, \\
& \text { then } k \leq 24 \cdot 2^{n-6} \text {. }
\end{aligned}
$$

The pairwise intersections in (3.29) are singletons, whereby the only way that the intersection in (3.30) is empty is that $\left|\left\{a_{1}, b_{1}, a_{2}, b_{2}, a_{3}, b_{3}\right\}\right|=3$. Hence, for all $1 \leq i<j \leq t$, we have that $U_{i} \cap U_{j}=U_{1} \cap U_{2} \cap U_{3}$ and $\left|U_{1} \cap U_{2} \cap U_{3}\right|=\left|U_{i} \cap U_{j}\right|=$ $2 \cdot 2^{n-6}$, and (3.30) follows from (3.22). We also claim that

if $t \geq 3,(3.29)$, and $\left\{a_{1}, b_{1}\right\} \cap\left\{a_{2}, b_{2}\right\} \cap\left\{a_{3}, b_{3}\right\} \neq \emptyset$, then $k \leq 25 \cdot 2^{n-6}$. 
With the assumption made in (3.31), if we consider the same intersections as in the argument right after (3.30), then we obtain that $\left|\left\{a_{1}, b_{1}, a_{2}, b_{2}, a_{3}, b_{3}\right\}\right|=4$. Hence, $\left|U_{i} \cap U_{j}\right|=2 \cdot 2^{n-6}$ and $\left|U_{1} \cap U_{2} \cap U_{3}\right|=1 \cdot 2^{n-6}$, and (3.31) follows from (3.22). Our next observation is that

$$
\text { if } t \leq 2 \text { and (3.29), then } k \geq 26 \cdot 2^{n-6} \text {. }
$$

For $t \leq 1$, this is clear from Theorem 2.3(i), Lemma 3.4, and Theorem 2.3(ii); so let $t=2$. Since the intersection in (3.29) is a singleton, the two ubt-antichains are of the form $\{a, b\}$ and $\{c, b\}$. Since $\{a, c\}$ cannot be a third ubt-antichain, the elements $a$ and $c$ are comparable, whereby Lemma 3.5, and Theorem 2.3(iii) imply that $k=26 \cdot 2^{n-6}$. Thus, (3.32) holds. Now, the required $k \leq 25 \cdot 2^{n-6}$ follows from (3.30), (3.31), (3.32), and the paragraph above (3.29); completing the first part of the proof.

In the rest of the proof, we will always assume that $k=25 \cdot 2^{n-6}$, even if this is not emphasized all the time. We claim that

$$
\begin{aligned}
& \text { if } k=25 \cdot 2^{n-6} \text { and } t \geq 3 \text {, then } t=3, v:=v_{1}=\cdots=v_{t} \text {, } \\
& \text { and (3.26) holds for all } 1 \leq i<j \leq t .
\end{aligned}
$$

Assuming the premise of (3.33), we obtain from (3.7) that the size of the set $\left\{a_{i}, b_{i}, v_{i}, a_{j}, b_{j}, v_{j}\right\}$ is not 6 . We obtain from (3.17) that it is neither 5 , whereby this size is 4 since $\left\{a_{i}, b_{i}\right\} \neq\left\{a_{j}, b_{j}\right\}$. Thus, (3.15) implies $v:=v_{1}=\cdots=v_{t}$ as well as the validity of $(3.26)$. The $\left|\left\{a_{i}, b_{i}\right\} \cap\left\{a_{j}, b_{j}\right\}\right|=1$ part of $(3.26)$ implies that apart from notation (that is, modulo permutations of the sets $\{i, j, m\},\left\{a_{i}, b_{i}\right\}$, $\left.\left\{a_{j}, b_{j}\right\},\left\{a_{m}, b_{m}\right\}\right)$,

whenever $1 \leq i<j<m \leq t$, then either $b_{i}=a_{j}, b_{j}=a_{m}$, and $b_{m}=a_{i}$, or $b:=b_{i}=b_{j}=b_{m}$ and $\left|\left\{a_{i}, a_{j}, a_{m}\right\}\right|=3$.

It follows similarly to (3.22) and (3.23) that

if the first alternative of (3.34) holds, then $\left|U_{i} \cup U_{j} \cup U_{m}\right|=$ $((4+4+4)-(2+2+2)+2) \cdot 2^{n-6}$, whereby $k \leq(32-8) \cdot 2^{n-6}$, which contradicts $k=25 \cdot 2^{n-6}$,

since $U_{i} \cap U_{j} \cap U_{m}=U_{i} \cap U_{j}$. Thus, (3.35) excludes the first alternative of (3.34). Hence we have the second alternative $\left|U_{i} \cap U_{j} \cap U_{m}\right|=2^{n-6}$, and it follows similarly to $(3.22)$ and (3.23) that

$$
\left|U_{i} \cup U_{j} \cup U_{m}\right|=((4+4+4)-(2+2+2)+1) \cdot 2^{n-6}=7 \cdot 2^{n-6} .
$$

Now, for the sake of contradiction, suppose that $t \geq 4$. Then we can pick an index $s \in\{1, \ldots, t\} \backslash\{i, j, m\}$. The ubt-antichain $\left\{a_{s}, b_{s}\right\}$ belongs to $U_{s}$ but it does not belong to $U_{i}$ since the members of $U_{i}$ contain both $a_{i}$ and $b_{i}$ but $\left\{a_{s}, b_{s}\right\} \neq\left\{a_{i}, b_{i}\right\}$. Similarly, $\left\{a_{s}, b_{s}\right\}$ belongs neither to $U_{j}$, nor to $U_{m}$, whence it is not in $U_{i} \cup U_{j} \cup U_{m}$. Hence, $U_{i} \cup U_{j} \cup U_{m}$ is a proper subset of $U_{i} \cup U_{j} \cup U_{m} \cup U_{s}$, which is disjoint from $\operatorname{Sub}\left(S^{+} ; \vee\right)$ by (3.10). Thus, by (3.36), strictly more than $7 \cdot 2^{n-6}$ subsets of $S^{+}$ are not in $\operatorname{Sub}\left(S^{+} ; \vee\right)$, and we obtain that $k=\left|\operatorname{Sub}\left(S^{+} ; \vee\right)\right|<(32-7) \cdot 2^{n-6}$. This contradicts $k=25 \cdot 2^{n-6}$ and excludes that $t \geq 4$. Thus, $t=3$ and we have proved (3.33).

Next, assume that $t \geq 3$. We know from (3.33) that $t=3$. Furthermore, we have by (3.33), (3.34), and (3.35) that $\left\{a_{1}, b\right\},\left\{a_{2}, b\right\}$, and $\left\{a_{3}, b\right\}$ is the list of all ubt-antichains of $\langle S ; \wedge\rangle$ and they have a common join $v$. No two of $a_{1}, a_{2}$, and $a_{3}$ are incomparable, since otherwise those two would form a ubt-antichain (with 
upper bound $v$ ). Hence, we can assume that $a_{1}<a_{2}<a_{3}$. Thus, it follows from Lemma 3.7 that $\langle S ; \wedge\rangle$ is a quasi-tree semilattice with nucleus $N_{6}$.

Finally, assume that $t \nsupseteq 3$. By Theorem 2.3(i)-(ii) and Lemma 3.4, $t \notin\{0,1\}$, whence $t=2$. There are several cases to consider.

Case 1 (we assume that $v_{1}=v_{2}$ and $\left\{a_{1}, b_{1}\right\} \cap\left\{a_{2}, b_{2}\right\} \neq \emptyset$ ). By the $a-b$ symmetry, we can choose the notation so that $a:=a_{1}, b:=b_{1}=b_{2}$, and $c:=a_{2}$. If $a \| c$, then $\{a, c\}$ is a third ubt-antichain (with upper bound $v_{1}=v_{2}$ ), contradicting $t=2$. Hence, we can assume that $a<c$. But then, by Lemma 3.5, $\langle S ; \wedge\rangle$ is a quasi-tree semilattice with nucleus $N_{5}$, and so Theorem 2.3 (iii) gives that $k=26 \cdot 2^{n-6}$, a contradiction again since $k=25 \cdot 2^{n-6}$ has been assumed. So Case 1 cannot occur.

Case 2 (we assume that $v_{1}=v_{2}$ and $\left\{a_{1}, b_{1}\right\} \cap\left\{a_{2}, b_{2}\right\}=\emptyset$ ). Observe that for every $X \subseteq\left\{a_{1}, b_{1}, a_{2}, b_{2}\right\}$ such that $|X|=2$,

$$
\text { if }\left\{a_{1}, b_{1}\right\} \neq X \neq\left\{a_{2}, b_{2}\right\} \text {, then } X \text { is not an antichain, }
$$

since otherwise $X$ would be a third ubt-antichain with upper bound $v_{1}=v_{2}$. By the 1-2 symmetry, we can assume that $a_{1}<a_{2}$. By (3.37), $a_{2}$ and $b_{1}$ are comparable elements. If we had that $a_{2} \leq b_{1}$, then we would obtain $a_{1} \leq b_{1}$ by transitivity, contradicting that $\left\{a_{1}, b_{1}\right\}$ is a ubt-antichain. Hence, $b_{1}<a_{2}$. But then the inequality $v_{1}=a_{1} \vee b_{1} \leq a_{2}<v_{2}=v_{1}$ is a contradiction. Therefore, Case 2 cannot occur either.

Cases 1 and 2 make it clear that now, when $t=2$, we have that $v_{1} \neq v_{2}$. We obtain from (3.7) and (3.15) that

$$
\left|\left\{a_{1}, b_{1}, v_{1}, a_{2}, b_{2}, v_{2}\right\}\right|=5 .
$$

The following two cases have to be considered.

Case 3 (we assume that $v_{1} \neq v_{2}$ and $\left\{a_{1}, b_{1}, a_{2}, b_{2}\right\} \cap\left\{v_{1}, v_{2}\right\}=\emptyset$ ). This assumption and (3.38) allow us to assume that $\left\{a_{1}, b_{1}\right\}=\{a, b\}$ and $\left\{a_{2}, b_{2}\right\}=\{c, b\}$. So $v_{1}=a \vee b$ and $v_{2}=c \vee b$. For the sake of contradiction, suppose that $a$ and $c$ are comparable. Let, say, $a<c$; then $v_{1}=a \vee b \leq c \vee b=v_{2}$. But $v_{1} \neq v_{2}$, so $v_{1}<v_{2}$. If we had that $c \leq v_{1}$, then $v_{2}=b \vee c \leq v_{1}$ would contradict $v_{1}<v_{2}$. If we had that $v_{1} \leq c$, then this would lead to the contradiction $b \leq c$ by transitivity. Hence, $c \| v_{1}$. So $\left\{c, v_{1}\right\}$ is an additional ubt-antichain (with upper bound $v_{2}$ ), which is a contradiction showing that $a \| c$. If $v_{1}$ and $v_{2}$ were comparable, then the larger one of them would be an upper bound of $\{a, c\}$, and so $\{a, c\}$ would be a third ubt-antichain. Thus, $v_{1} \| v_{2}$, and Lemma 3.6 gives that $\langle S ; \wedge\rangle$ is a quasi-tree semilattice with nucleus $F$, as required.

Case 4 (we assume that $v_{1} \neq v_{2}$ and $\left\{a_{1}, b_{1}, a_{2}, b_{2}\right\} \cap\left\{v_{1}, v_{2}\right\} \neq \emptyset$ ). Since $a$ and $b$ play symmetric roles and so do the subscripts 1 and 2 , we can assume that $v_{1}=a_{2}$. We have that $\left|\left\{a_{1}, b_{1}, a_{2}, b_{2}\right\}\right|=4$ since $b_{2} \not \leq a_{2}=v_{1}$ excludes the possibility that $b_{2} \in\left\{a_{1}, b_{1}, a_{2}\right\}$. None of the sets $\left\{a_{1}, b_{2}\right\}$ and $\left\{b_{1}, b_{2}\right\}$ is an antichain, since otherwise the set in question would be a new ubt-antichain with upper bound $v_{2}$, which would be a contradiction. Hence, $a_{1}$ and $b_{2}$ are comparable elements, and so are $b_{1}$ and $b_{2}$. If we had that $a_{1} \geq b_{2}$ or $b_{1} \geq b_{2}$, then transitivity would lead to $a_{2}=v_{1} \geq b_{2}$, a contradiction. Thus, $a_{1} \leq b_{2}$ and $b_{1} \leq b_{2}$. But then $a_{2}=v_{1}=a_{1} \vee b_{1} \leq b_{2}$ is a contradiction. This shows that Case 4 cannot occur. 
Now that all cases have been considered, we have shown that if $k=25 \cdot 2^{n-6}$, then $\langle S ; \wedge\rangle$ is of the required form.

Finally, if $\langle S ; \wedge\rangle$ is a quasi-tree semilattice with nucleus $N_{6}$, then using the Inclusion-Exclusion Principle and (3.10), a computation similar to (3.22) and (3.23) yields that

$$
|\operatorname{Con}(S ; \wedge)|=2^{n-6}(32-(4+4+4)+(2+2+2)-1)=25 \cdot 2^{n-6},
$$

as required. Also, if the nucleus is $F$, then a computation similar to (3.11)-(3.13) derives from (3.10) and the Inclusion-Exclusion Principle that

$$
|\operatorname{Con}(S ; \wedge)|=2^{n-6}(32-(4+4)+1)=25 \cdot 2^{n-6} .
$$

This completes the proof of Theorem 2.3(iv).

\section{REFERENCES}

[1] Czédli, G.: A note on lattices with many congruences. Acta Universitatis Matthiae Belii, Series Mathematics, Online (2018), 22-28; http://actamath.savbb.sk/pdf/oacta2018003.pdf

[2] Czédli, G., Mureşan, C.: On principal congruences and the number of congruences of a lattice with more ideals than filters. Acta Sci. Math. (Szeged) (submitted), http://arxiv.org/abs/1711.06394

[3] Freese, R.: Computing congruence lattices of finite lattices. Proc. Amer. Math. Soc. 125, 3457-3463, 1997

[4] Freese, R.; Nation, J.B.: Congruence lattices of semilattices. Pacific Journal of Mathematics 49 (1973), 51-58.

[5] Górnicka, A., Grygiel, J., Tyrala I.: On the lattice of tolerances for a finite chain. Scientific Issues, Jan Długosz University in Czȩstochowa, Mathematics 21, 25-30 (2016) DOI http://dx.doi.org/10.16926/m.2016.21.03

[6] Grätzer, G.: Lattice Theory: Foundation. Birkhäuser, Basel (2011)

[7] Grätzer, G.: The Congruences of a Finite Lattice, A Proof-by-Picture Approach, second edition. Birkhäuser, 2016

[8] Kulin, J., Mureşan, C.: Some extremal values of the number of congruences of a finite lattice. https://arxiv.org/abs/1801.05282

[9] Mureşan, C: Cancelling congruences of lattices while keeping their filters and ideals. https://arxiv.org/abs/1710.10183

[10] Szpilrajn, E.: Sur l'extension de l'ordre partiel. Fundamenta Mathematicae 16, 386-389, (1930)

E-mail address: czedli@math.u-szeged.hu

URL: http://www.math.u-szeged.hu/ czedli/

Bolyai Institute, University of Szeged, Hungary 6720 